\title{
Síndrome de Sturge-Weber: revisión de la literatura
}

\author{
Sturge-Weber syndrome: literature review
}

Sanz-Arrazola Heydi Yvana ${ }^{1}$, Antezana-Llaveta Gabriela ${ }^{2}$

\section{Resumen}

El síndrome de Sturge-Weber es un trastorno neurocutáneo, congénito, esporádico e infrecuente que afecta aproximadamente a 1 de cada 20000 a 500000 nacidos vivos y que se relaciona con una mutación genética activadora somática en GNAQ. Clínicamente se caracteriza por la presencia de una mácula en vino de Oporto en la piel de territorio trigeminal, angiomatosis leptomeníngea y glaucoma. Puede asociarse a diferentes manifestaciones clínicas, de las cuales las crisis epilépticas representan la manifestación neurológica más frecuente que se asocia a un deterioro cognitivo importante en estos pacientes. En el presente artículo se realiza una revisión descriptiva de la literatura sobre los aspectos etiológicos, fisiopatológicos, de clasificación, clínicos, diagnósticos y del tratamiento del síndrome de Sturge-Weber.

Palabras claves: Síndrome de Sturge-Weber, angiomatosis, leptomeníngea, crisis epiléptica.

\section{Abstract}

Sturge-Weber syndrome is a rare, sporadic, congenital neurocutaneous disorder affecting approximately 1 in 20,000 to 50,000 live births that is associated with a somatic activating gene mutation in GNAQ. Clinically it is characterized by the presence of a port wine stain on the skin of trigeminal territory, leptomeningeal angiomatosis and glaucoma. It can be associated with different clinical manifestations, of which the epileptic seizures represents the most frequent neurological manifestation associated with significant cognitive impairment in these patients. This article makes a descriptive review of the literature on the etiological, pathophysiological, classification, clinical, diagnostic and treatment aspects of Sturge-Weber syndrome.

Keywords: Sturge-Weber syndrome, angiomatosis, leptomeningeal, epileptic seizures.

$\mathrm{E}$ l síndrome de Sturge-Weber (SSW), conocido también como angiomatosis encefalotrigeminal, pertenece a un grupo de trastornos llamados facomatosis, síndromes neurocutáneos que involucran los sistemas nervioso central y cutáneo ${ }^{1}$. Corresponde al tercer trastorno neurocutáneo más común, después de la neurofibromatosis y la esclerosis tuberosa, afectando aproximadamente a 1 de cada 20000 a 50.000 nacidos vivos ${ }^{2,3}$.

El SSW es esporádico e infrecuente, no tiene distinción de raza ni género y generalmente es de carácter no hereditario ${ }^{4}$. Clínicamente se caracteriza por la presencia de un angioma facial que se observa como una mácula en vino de Oporto localizado en territorio trigeminal, angiomatosis leptomeníngea y glaucoma, aunque también se han descrito alteraciones en la vía oral y respiratoria ${ }^{3,4,5}$.

El angioma facial es evidente desde el nacimiento en la gran mayoría de los casos, y se estima que "un recién nacido con una mácula en vino de Oporto en la frente o en el párpado superior, tiene un riesgo de 10 a $35 \%$ de presentar compromiso cerebral", sin embargo puede no estar presente, por lo cual es importante reconocer las otras manifestaciones

${ }^{1}$ M.D.-Médico Neuropediatra. Hospital del Niño Manuel Ascencio Villarroel. Cochabamba, Bolivia. https://orcid.org/0000-0001-8455-4729.

${ }^{2}$ Estudiante de Medicina, Facultad de Medicina - Universidad Mayor de San Simón. Cochabamba, Bolivia. https://orcid.org/0000-0002-2485-3425.

${ }^{*}$ Correspondencia a: Gabriela Antezana Llaveta

Correo electrónico: gabriela_antezana@hotmail.com

Recibido el 12 de agosto de 2020. Aceptado el 24 de octubre de 2020. de este trastorno, como lo son las crisis epilépticas, las cuales se presentan como la manifestación neurológica más frecuente y destacables que se desarrolla sobre todo en los primeros meses de vida ${ }^{3,4}$. Las crisis pueden a empeorar si no son tratadas adecuadamente, haciéndose refractarias, provocando deterioro motor y/o grave retraso en el lenguaje y desarrollo ${ }^{4}$. Se ha descrito una asociación de este síndrome con una mutación en el gen GNAQ, esta mutación afecta la vía de señalización MAPK-ERK, lo que resulta en anomalías endoteliales y malformaciones de los vasos sanguíneos ${ }^{6}$.

En el presente artículo se realiza una revisión de carácter descriptivo de los aspectos etiológicos, fisiopatológicos, de clasificación, clínicos, diagnósticos y del tratamiento del SSW.

\section{Revisión bibliográfica}

Para la realización del presente artículo se llevó a cabo una búsqueda sistemática y metódica de fuentes primarias de información en buscadores online e indizaciones científicas, tales como: PubMed, ElSevier, Scielo, MedLine, etc.

Las fuentes primarias corresponden a artículos científicos publicados (Review Articule, Case Report, Serie de Casos y Ensayos Clínicos) que serán mencionados en las referencias bibliográficas.

\section{Desarrollo y discusión del tema}

Etiología

Matthew y col. en el año 2013 encontraron en su estudio que el SSW y las máculas en vino de Oporto son causados 
por una mutación activadora somática en GNAQ (c.548G>A; p.R183Q), tras realizar la secuenciación genómica en muestras de tejidos de lesiones cutáneas y cerebrales en pacientes con SSW. En el año 2017 Huang y col. encontraron hallazgos similares en pacientes con SSW, en este caso el tipo de muestra estudiada fue de células endoteliales de tejido nervioso afectado, encontrando la mutación GNAQ p.R183Q en las mismas ${ }^{6,7}$. GNAQ codifica Gaq, que corresponde a la subunidad alfa de las proteínas $G$ heterotriméricas ${ }^{4,7}$. Las proteínas $\mathrm{G}$ son un grupo de proteínas transmembranales que cumplen una función en la transducción celular al trasmitir la señalización desde los receptores acoplados a proteína $G$ en la superficie celular hasta el interior de la célula; estas proteínas reciben su nombre debido a que están asociadas a GTP (Guanosina TriFosfato) y se dice que tienen actividad GTPasa porque son capaces de hidrolizar una molécula de GTP a GDP (Guanosina DiFosfato), en el proceso de transducción ${ }^{7,8}$. Es probable que las mutaciones en GNAQ induzcan cambios en la morfología celular y el crecimiento celular principalmente mediante la regulación a la alza de la vía de señalización MAPK-ERK, lo que proporciona una base de patogénesis molecular en el SSW; también se cree que una mutación activadora somática en GNAQ podría tener potencial oncogénico, de hecho las mutaciones somáticas de GNAQ en melanocitos se han asociado con el melanoma uveal en una mutación activadora que conduce a un aumento de la señalización cuesta abajo a través de la vía MAPK, sin embargo los melanomas poseen varias mutaciones aparte de GNAQ que hay que tomar en cuenta y en el SSW no se encontraron mutaciones acumulativas en las muestras estudiadas ${ }^{6,9,10}$.

Se ha planteado la hipótesis de que durante los períodos vulnerables del desarrollo embrionario, la señalización inicial moderadamente aumentada cuesta abajo de $\mathrm{G} \alpha \mathrm{q}$, o la señalización desregulada a través de receptores acoplados a proteína $\mathrm{G}$ como desregulación de la señalización vascular MAPK y/o PI3K, podría dar lugar a vasos sanguíneos malformados, dilatados e inervados anormalmente como hallazgos subyacentes a la mácula en vino de Oporto y en el SSW $^{6,11,12}$.

\section{Fisiopatología}

Las alteraciones primarias en la vasculatura en el SSW corresponden a una proliferación de pericitos y duplicación de las membranas basales sin ectasia significativa, que ocurren antes de la dilatación de vasos sanguíneos. A pesar de que los hallazgos angiopáticos habrían sido descritos como una dilatación de las vénulas poscapilares, en la actualidad se sugiere cambiar esta descripción por otra que encaje mejor con los hallazgos actuales a propósito del mismo. Por ejemplo se sabe que los vasos sanguíneos del SSW coexpresan marcadores de células madre, CD133 y CD166, así como el marcador venoso EphB1 y el marcador arterial EfnB2, y que durante el desarrollo, tanto las arteriolas dérmicas como las vénulas se diferencian de un plexo capilar primario y de forma predeterminada, se cree que este plexo capilar primario se convierte en una vena con una expresión consistente de EphB1. Es por eso que se sugiere cambiar la descripción de una "dilatación de vénulas poscapilares" por el de una "dilatación progresiva de vasculatura similar a vénulas"11,13.

La mayoría de las lesiones de SSW son unilaterales con una distribución típica del dermatoma del trigémino, existe generalmente una angiomatosis intracraneal leptomeníngea que suele ser ipsilateral a la mácula en vino de Oporto, aunque también puede ser bilateral, afectando el lóbulo occipital, occipitoparietal y en ocasiones a todo un hemisferio cerebral; los vasos afectados suelen mostrarse tortuosos y dilatados, además muestran sobreexpresión de la glicoproteína fibronectina y del factor de crecimiento endotelial vascular (VEFG) con aumento de proliferación celular y apoptosis ${ }^{3}$. Diversos estudios han demostrado además atrofia y/o calcificación de la corteza cerebral, que podría tratarse de "calcificaciones distróficas debidas a hipoxia", ya que en una convulsión el flujo sanguíneo cerebral puede mermar hasta niveles isquémicos ${ }^{3,4}$. Una crisis epiléptica por lo general ocurre cuando hay un desequilibrio entre la excitación y la inhibición en una o más áreas del cerebro; Juhász y col. en un estudio donde utilizaron imágenes espectroscópicas de resonancia magnética de protones (MRSI) determinaron que las concentraciones de glutamato (un neurotransmisor excitatorio) aumentaban en el hemisferio afectado por esta patología, por tanto se sostiene que la estimulación excesiva de los receptores de glutamato en el tejido cerebral afectado está relacionado con las crisis epilépticas al provocar una "lesión cerebral excitotóxica", y que los niveles de glutamato podrían encontrarse en altas concentraciones debido a la hipoxia ${ }^{3,14,15}$.

Los pacientes con SSW pueden desarrollar angiomatosis del plexo coroideo y glaucoma durante la evolución de la enfermedad, estas lesiones tienden a ser casi siempre ipsilaterales a la mácula en vino de Oporto y el riesgo es mayor cuando esta alteración afecta a ambos párpados; en estos casos el riesgo del glaucoma puede alcanzar hasta el $50 \%$ de los pacientes, estos desarrollan glaucoma sobre todo en la infancia debido a anomalías de la cámara anterior y en la edad adulta temprana debido a una elevación epiescleral de la presión venosa, posiblemente debido a la presencia de hemangioma epiescleral y derivaciones arteriovenosas ${ }^{4,16}$.

\section{Clasificación}

Roach habría clasificado el SSW en tres tipos, en relación a si la afectación comprende varios tejidos o son lesiones aisladas:

- Tipo I: El paciente presenta angioma facial, leptomeníngeo y puede tener glaucoma.

- Tipo II: El paciente presenta angioma facial pero no leptomeníngeo y puede tener glaucoma.

- Tipo III: El paciente presenta angiomas leptomeníngeo aislados pero no presenta glaucoma ${ }^{17}$.

También se lo ha clasificado al SSW como completo cuando el paciente presenta tanto angiomas faciales como leptomeníngeos, e incompleto cuando solo afecta a un tejido $\mathrm{u}$ otro ${ }^{4,17}$. 

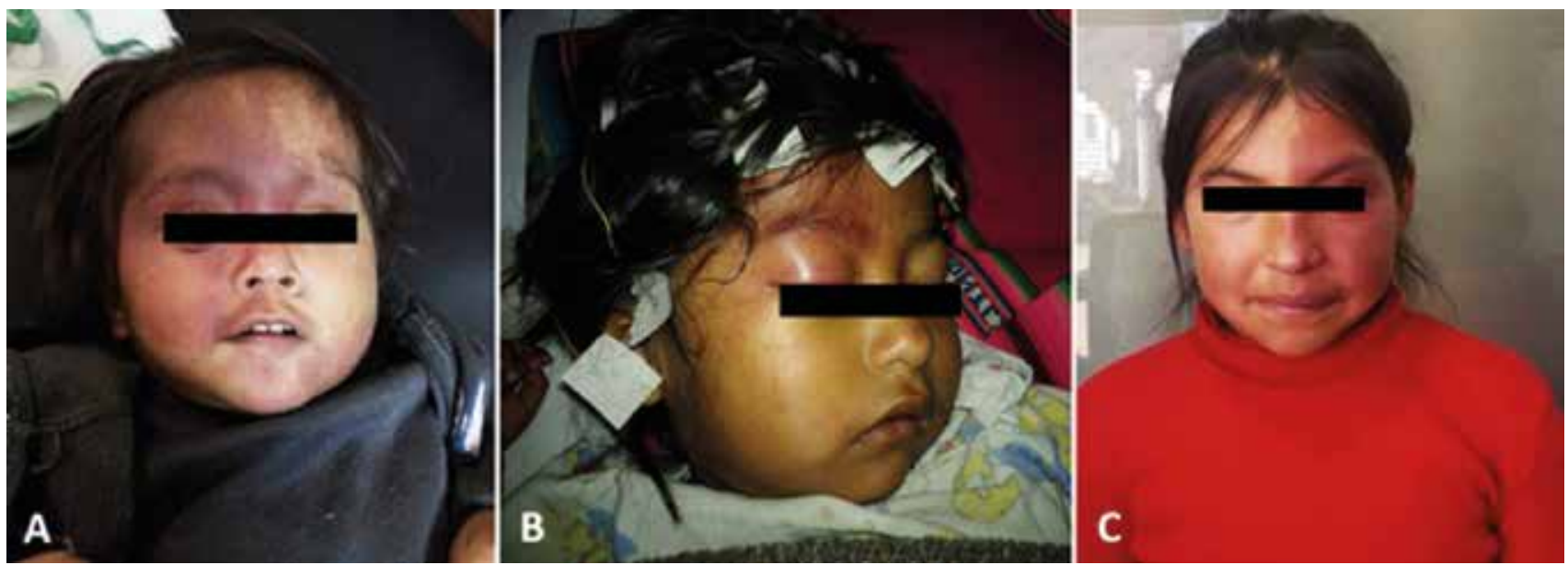

Figura 1. Mácula en vino de Oporto, con afectación bilateral y afectación extensa en hemicara derecha en territorio trigeminal (A). Mácula en vino de Oporto con afectación del territorio de rama oftálmica-trigeminal (B). Mácula en vino de Oporto en hemicara izquierda en territorio trigeminal, con signos leves de hipertrofia del labio superior en hemicara izquierda (C).

Fuente: elaboración propia.

\section{Aspectos Clínicos}

El SSW puede tener diversas manifestaciones clínicas como ser manifestaciones cutáneas, neurológicas, oculares y endocrinas.

\section{Manifestaciones cutáneas}

La mácula en vino de Oporto también llamada nevus flammeus es la manifestación clínica cutánea más común, suele ser unilateral, típicamente ha sido descrita en la distribución de la rama oftálmica y maxilar del trigémino, aunque actualmente se tiene más evidencia de que esta lesión tiene una distribución embriológica vascular y no tanto el de una inervación neural de la cara; es de un color que oscila desde rosa pálido a púrpura, que puede extenderse al cuello u otras partes del cuerpo y estar presente desde el nacimiento (Ver figura 1); se ha asociado la localización de esta malformación con el riesgo de desarrollar angiomas leptomeníngeos y glaucoma, siendo mayor el riesgo cuando la mácula en vino de Oporto se localiza en la región frontal en relación a los que se encuentran localizados en la región inferior de la cara, además existe evidencia de que a mayor tamaño de la lesión, existiría una mayor gravedad de la afectación neurológica ${ }^{1,3,4,18}$.

Las malformaciones vasculares si no se tratan pueden progresar y desarrollar hipertrofia de los tejidos blandos, del tejido óseo o formación de nódulos de las estructuras involucradas. Puede ocurrir una hipertrofia del hueso maxilar superior o de la mandíbula que puede causar una mala oclusión dental; en general los cambios hipertróficos tienen una pobre respuesta al tratamiento con láser y pueden llevar a una deformidad facial significativa que requiera tratamiento quirúrgico ${ }^{19}$. La edad media de presentación de estas alteraciones son a los nueve años, y el hueso maxilar superior es el que presenta con mayor frecuencia datos de hipertrofia ${ }^{3}$.

\section{Manifestaciones neurológicas}

Las manifestaciones neurológicas más importantes de la angiomatosis leptomeníngea son las crisis epilépticas, el retraso en el neurodesarrollo, hemiparesias lentamente progresivas, cefaleas vasculares de tipo migrañoso y episodios similares a eventos cerebrovasculares también llamados episodios stroke$l_{i k e^{3}}$. Las crisis epilépticas, la atrofia de la corteza cerebral, el retraso en el desarrollo y las deficiencias intelectuales ocurren comúnmente en la infancia y estas pueden empeorar con la edad $^{3,11}$.

Las crisis epilépticas suelen ser el primer síntoma neurológico del SSW, desarrollándose hasta en el $80 \%$ de estos pacientes, iniciándose en la infancia temprana; sin embargo pueden producirse a cualquier edad. Se ha descrito que los pacientes que inician con las crisis antes de los 2 años de edad, presentan crisis refractarias y difíciles de controlar con un mayor compromiso neurológico ${ }^{1,3,4}$. Las crisis epilépticas suelen empezar como crisis focales que posteriormente evolucionan a crisis tónico clónicas generalizadas, es posible encontrar crisis como espasmos infantiles, crisis mioclónicas o atónicas ${ }^{4}$. Estas crisis pueden ser precipitadas por episodios febriles ${ }^{4}$. El deterioro neurológico es progresivo y está directamente relacionado con la frecuencia y duración de las crisis epilépticas, está asociado a una alteración de la perfusión cerebral como isquemia cerebral ${ }^{3}$. Las crisis prolongadas, sobre todo en lactantes y niños pequeños también pueden desencadenar un accidente cerebrovascular (ACV) ${ }^{4}$.

Los episodios stroke-like pueden causar déficits neurológicos transitorios, pueden existir hemiparesias que se desarrollan de forma aguda o con el inicio de las crisis, la extremidad afectada no crece al mismo ritmo que el resto del cuerpo, por lo que aparecen hemiatrofias en las extremidades afectadas ${ }^{4}$.

Se han descrito problemas del comportamiento con mayor prevalencia en los pacientes con SSW, como por ejemplo los trastornos del espectro autista (TEA), trastorno por déficit de atención con hiperactividad (TDAH) y trastorno de oposición desafiante; además la depresión es más prevalente en este 
grupo de pacientes, y también se han descrito algunos síntomas psicóticos como ser alucinaciones visuales y auditivas ${ }^{4,20}$.

Los pacientes con SSW pueden crecer normalmente durante los primeros meses de vida, sin embargo posteriormente la tendencia es presentar un retraso en el desarrollo ${ }^{4}$.

\section{Manifestaciones oculares}

La malformación vascular en el ojo puede afectar la conjuntiva, la epiesclera, la retina y/o la coroides ${ }^{3}$.

Los pacientes pueden desarrollar glaucoma, como una de las manifestaciones oculares más frecuentes, suele ser unilateral a la mácula en vino de Oporto pero no siempre es así, resulta como consecuencia de una presión venosa epiescleral elevada que ocasiona un incremento de la presión intraocular ${ }^{21}$. Sin embargo, se describen dos formas de glaucoma en SSW, la primera representa al 60\%, también llamada congénita y está asociado a anomalías en la cámara anterior del ojo, y la segunda forma también llamada tardía representa el $40 \%$ y esta se relaciona con una elevada presión venosa epiescleral ${ }^{3}$.

El hemangioma coroideo, puede estar presente como una forma circunscrita o difusa, son típicamente unilaterales e ipsilaterales a la macula en vino de Oporto, pero este dato tampoco siempre se cumple; los hemangiomas coroideos bilaterales son $\operatorname{raros}^{3,16}$. La coroides de los pacientes con SSW se engrosa en la adolescencia y edad adulta, y es en este periodo cuando pueden aparecer complicaciones como hemorragia subretiniana, desprendimiento de retina seroso, edema macular cistoide y desprendimiento del neuroepitelio macular llegando a provocar incluso una pérdida grave de la visión ${ }^{16}$.

Pueden existir alteraciones del campo visual en los pacientes con SSW, como una hemianopsia homónima que puede deberse a angiomas que afectan ambos lóbulos occipitales o que involucran las vías ópticas. ${ }^{4}$ Otras anomalías oculares menos frecuentes pero que también han sido descritas incluyen desprendimiento de retina, heterocromía del iris, estrabismo, luxación del cristalino, neovascularización del iris y la coroides ${ }^{3}$.

\section{Manifestaciones endocrinas}

Se ha descrito que las células secretoras de la hormona de crecimiento de la glándula pituitaria son particularmente sensibles al insulto vascular, lo que puede condicionar a un mayor riesgo de alteraciones endocrinas a este nivel, también se han descrito casos de hipopituitarismo central en el SSW en este contexto ${ }^{22,23}$.

\section{Diagnóstico}

Se puede diagnosticar mediante el hallazgo de síntomas clínicos típicos y por la apariencia facial de la mácula en vino de Oporto" ${ }^{11}$. "Todos los pacientes con nevus facial deben someterse a una evaluación radiológica para descartar SSW", esta evaluación puede ser de ayuda para detectar las calcificaciones intracraneales clásicas de estos pacientes ${ }^{1}$ (Figura 2). Se han visto sin embargo múltiples hallazgos radiológicos como ser atrofia de lóbulos cerebrales, hipertrofia de plexos coroideos, agrandamiento de las venas cerebrales profundas, anomalías de drenaje venoso y recientemente se ha descrito un agrandamiento asimétrico del seno cavernoso ${ }^{4,24}$ (Figura 3).

Las pruebas de imágenes, como ecografía doppler, tomografía computarizada (TC) o imágenes por resonancia magnética (RM), pueden ser útiles para determinar cualquier posible malformación vascular localizada en tejidos profundos, por ejemplo, malformaciones vasculares cerebrales $\mathrm{o}$ malformaciones arteriovenosas ${ }^{4,11}$. La técnica de neuroimagen de elección para el diagnóstico de SSW actualmente es la resonancia magnética con contraste de gadolinio, ya que puede demostrar la presencia de angiomatosis leptomeníngea y determinar el grado de afectación de las estructuras del cerebro ${ }^{4}$.

El electroencefalograma (EEG) evalúa la actividad epileptiformes y la disfunción cerebral; la actividad a menudo se atenúa o disminuye en el hemisferio afectado, este patrón de actividad puede ser detectada desde los primeros meses de vida del paciente haciéndose más evidente con la progresión de la atrofia cerebral ${ }^{4}$.

El SSW debe diagnosticarse de forma diferencial con respecto a los hemangiomas infantiles, que son los tumores benignos infantiles más frecuentes y que habitualmente involucionan con el tiempo; molecularmente las células endoteliales de los hemangiomas infantiles son positivas para Glut-1, pero las células endoteliales del SSW no lo son ${ }^{25}$. Otros diagnósticos diferenciales incluyen el síndrome de Rendu-Osler-Weber, el síndrome de Angio-osteodistrofia, el síndrome de Maffuci, la enfermedad de Von Hippel Lindau y el síndrome de Klippel Trenaumy-Weber ${ }^{17}$.

\section{Tratamiento}

El tratamiento del SSW está dirigido hacia la mácula en vino de Oporto, a las crisis epilépticas, las crisis migrañosas, los eventos stroke-like y el glaucoma.

El tratamiento de elección para la mácula en vino de Oporto es la terapia con láser colorante pulsado (PDL), el inicio temprano del tratamiento puede disminuir la progresión a hipertrofia tisular y complicaciones visuales, de vías respiratorias y de la deglución ${ }^{3,26}$. Se ha descrito que el grado de respuesta al láser es mayor en la región frontal a diferencia de la zona malar o del prolabio y en general se requieren entre 7 a 15 sesiones para lograr un aclaramiento de la lesión; el aclaramiento definitivo o completo no siempre es posible 3 . "El láser calienta la hemoglobina dentro de los vasos sanguíneos y los destruyen, evitando las estructuras circundantes de la piel", sin embargo la mácula puede llegar a regenerarse con el tiempo, requiriendo mayor número de sesiones; la resistencia terapéutica puede deberse a la regeneración y revascularización de los vasos coagulados ${ }^{3,4}$. Se han descrito ciertos medicamentos adyuvantes al tratamiento con PDL que podrían mejorar la eficacia del tratamiento, como por ejemplo el Imiquimod tópico y la Rapamicina ${ }^{27}$. La Rapamicina como tratamiento adyuvante al PDL se ha mostrado como un potencial agente antiangiogénico que 


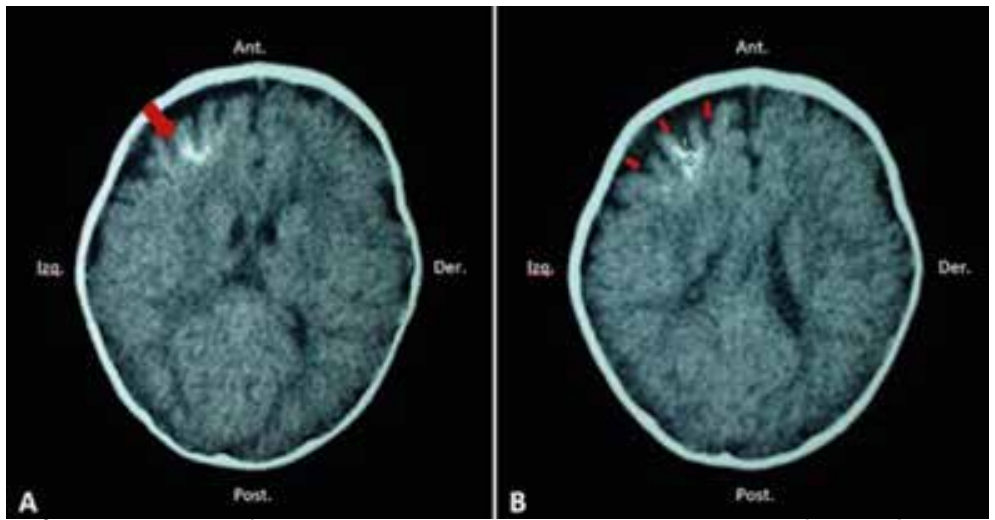

Figura 2. Corte axial de tomografía computarizada (TC) sin contraste en un paciente con síndrome de Sturge-Weber, donde se evidencia calcificación frontal izquierda de parénquima cerebral (flecha); estudio imagenológico corresponde al paciente de la figura 1-B (A). Se puede evidenciar una atrofia cortical frontal (flechas) (B).

Fuente: elaboración propia.

podría prevenir la revascularización después del tratamiento con PDL; sin embargo Greveling y col. recientemente en un ensayo aleatorio demostraron que la aplicación tópica de la solución disponible en el mercado (Rapamune 0,1\%) como un adyuvante al tratamiento PDL no parece mejorar el blanqueamiento de la lesión en el SSW, la Rapamicina oral podría tener resultados más prometedores, y se recomienda mayores investigaciones respecto a eficacia, efectos adversos y costo-efectividad ${ }^{28}$.

El objetivo principal del tratamiento neurológico es minimizar la actividad epiléptica con medicamentos antiepilépticos para evitar o minimizar el deterioro psicomotriz y otras alteraciones del neurodesarrollo en estos pacientes; es importante instruir a los padres e informarles sobre como reconocer estas crisis y cómo actuar una vez que estas ocurren, así como es importante recomendarles acerca de las enfermedades intercurrentes que cursen con fiebre para evitarlas en la medida en que sea posible ya que la fiebre puede desencadenar estas crisis; también se debe asegurar buena hidratación y oxigenación del paciente ${ }^{3}$. Se ha descrito que la mayoría de los pacientes logran controlar las crisis con 1 a 2 medicamentos antiepilépticos más la administración de ácido acetilsalicílico en dosis bajas ${ }^{4}$. Los medicamentos más usados en lactantes han sido la Oxcarbazepina, Carbamazepina, Levetiracetam y Fenobarbital y los pacientes con espasmos infantiles pueden responder al tratamiento con esteroides, Topiramato, Vigabatrina $o$ dieta cetogénica ${ }^{4}$. La Oxcarbazepina se asoció con menos efectos secundarios informados que otros antiepilépticos, por lo que puede ser preferible y se recomienda comenzar el tratamiento con Oxcarbazepina a menos que estén presentes características de crisis generalizadas ${ }^{29}$. El Topiramato a pesar de estar relacionado con desencadenar glaucoma agudo bilateral de ángulo cerrado empeorando el pronóstico en ciertos casos, no se asoció con signos relacionados al glaucoma en un análisis limitado de pacientes y los efectos secundarios informados del Valproato coincidieron con sus efectos conocidos, por lo que estos medicamentos podrían ser alternativas seguras para tratar la migraña $y$ la epilepsia concomitantes, además de los triptanes y la Lamotrigina que también se han utilizado para este fin $^{4,28}$. El ácido acetilsalicílico en dosis bajas (3 a $5 \mathrm{mg} / \mathrm{kg} /$ día) puede disminuir la frecuencia y la gravedad de los crisis epilépticas y de los episodios stroke-like ${ }^{30}$. Se ha planteado
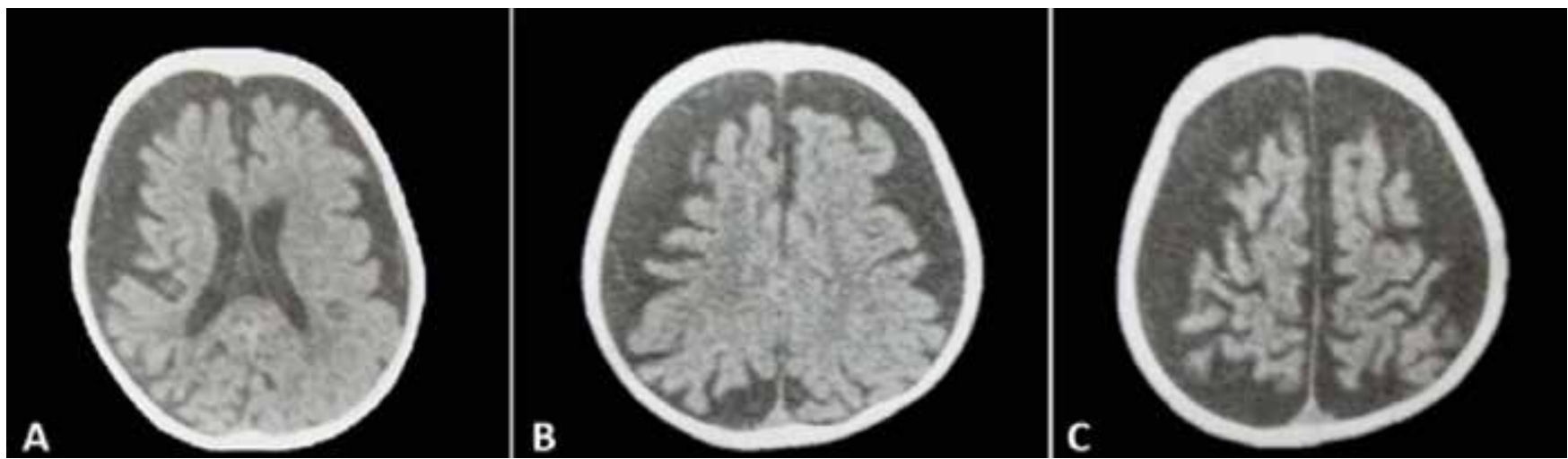

Figura 3. Tomografía computarizada (TC) sin contraste, correspondiente al paciente de la figura 1-A. Se evidencia corte axial que muestra atrofia cerebral difusa; coincidiendo con el retraso psicomotor que presenta este paciente con síndrome de Sturge-Weber.

Fuente: elaboración propia. 
un tratamiento presintomático sobre todo en pacientes con angiomatosis leptomeníngeas extensas bilaterales que tienen mayor riesgo de crisis graves y déficits neurológicos; en estos pacientes se ha utilizado Levetirazetam, Fenobarbital y otros antiepilépticos asociados a dosis bajas de ácido acetilsalicílico; no se recomienda dar el tratamiento presintomático a todos los pacientes con SSW por los efectos adversos de la medicación ${ }^{3,30}$. Se justifica un tratamiento agresivo en crisis epilépticas prolongadas y refractarias como medida terapéutica; y cuando el tratamiento médico falla, se considerará el tratamiento quirúrgico como ser la lesionectomía, bien por callostomía o hemisferectomía ${ }^{3,411}$. En relación al ácido acetilsalicílico se han descrito efectos adversos como hemorragias, gingivorragias, hematomas, sin embargo estos efectos adversos no se reportan con mayor gravedad 4 .

El objetivo principal en el tratamiento de glaucoma es disminuir la presión intraocular para evitar una lesión isquémica del nervio óptico ${ }^{3,4}$. El tratamiento con gotas oftálmicas para reducir la presión intraocular, como por ejemplo con prostaglandinas, betabloqueadores o inhibidores de la anhidrasa carbónica pueden ser eficaces porque disminuyen la producción de líquido en el ojo y aumentan el flujo de salida del humor acuoso a través de la vía uveoescleral; el Timolol y Lantaprost, han sido utilizados para el tratamiento de glaucoma en pacientes con $\mathrm{SSW}^{26,31}$. Si el paciente no responde al tratamiento médico se realizarán tratamientos quirúrgicos como la implementación de la válvula de glaucoma de Ahmed, y otros procedimientos invasivos; recientemente se ha descrito un caso de glaucoma tratado con éxito mediante el stent CyPass, este método ofrece un abordaje que es menos invasivo y se ha demostrado que ofrece una solución a largo plazo para reducir la presión intraocular en pacientes con SSW ${ }^{26}$.

\section{Discusión}

El SSW como vimos es un trastorno que se presenta de forma infrecuente en la población, y es de gran importancia poder reconocerla clínicamente; la presencia de la mácula en vino de Oporto debe alarmar a los padres y a los médicos por el riesgo de angiomatosis leptomeníngea y glaucoma más elevado que presentan estos pacientes en relación a la población general; esta lesión puede estar presente desde el nacimiento y es importante identificarlo adecuadamente, para poder dar lugar a una derivación a un especialista para el tratamiento de esta "marca de nacimiento", así como para la consulta con un neurólogo y oftalmólogo que realicen la evaluación y el tratamiento adecuado de la afectación cerebral u ocular (o ambos) si fuese necesario; en los casos en los que no presenten esta lesión probablemente el diagnóstico sea más difícil pero se hará guiado en las otras manifestaciones clínicas que pueden presentar estos pacientes. Es importante tomar en cuenta en el tratamiento el manejo multidisciplinario dirigido a tratar la enfermedad y sus complicaciones, así como brindar apoyo y soporte al paciente y a su familia; este equipo multidisciplinario además tendría que tener la función de hacer un seguimiento estrecho del paciente y sus complicaciones, de igual manera se considera de gran importancia no descuidar las manifestaciones emocionales y psicológicas de estos pacientes. Hasta la fecha no existe un tratamiento estandarizado para los pacientes con SSW, por tanto se debe siempre individualizar el tratamiento de cada paciente y se espera que en un futuro nazcan nuevas investigaciones en el tratamiento dirigidos sobre todo a la parte molecular de la etiología de este síndrome y que pueda brindar una esperanza a estos pacientes, para tener un mejor pronóstico en general.

Potencial conflicto de intereses: Los autores declaran no tener conflicto de intereses pertinentes a la presentación de este artículo.

\section{Referencias bibliográficas}

1. Saeed M.A., Hilal K., Chand P. Bilateral intracranial calcifications with bilateral facial cutaneous naevus: Sturge Weber syndrome. BMJ Case Rep [Internet]. 2017 [Citado 6 Sep 2020]; bcr2017219985. Disponible en:

https://doi.org/10.1136/bcr-2017-219985

2. Comi A.M. Chapter 11-Sturge-Weber syndrome. Handb Clin Neurol [Internet]. 2015 [Citado 6 Sep 2020]; 137(3): 157-68. Disponible en: https://doi.org/10.1016/B978-0-444-627025.00011-1

3. Higueros E., Roe E., Granell E., Baselga E. Síndrome de Sturge-Weber: revisión. Actas Dermosifiliogr [Internet]. 2017 [Citado 6 Sep 2020]; 108(5): 407-17. Disponible en: https:// www.actasdermo.org/es-pdf-S0001731016304422

4. Velázquez-Gallego C., Ceballos-Ruiz J.F., RuizJaramillo N., Villamizar-Londoño C. Rev Ecuat Neurol [Internet]. 2019 [Citado 6 Sep 2020]; 28(2): 105-14. Disponible en: http://revecuatneurol. com/wp-content/uploads/2019/10/2631-2581rneuro-28-02-000105.pdf
5. Nidhi C., Anuj C. Sturge Weber Syndrome: An Unusual Case with Multisystem Manifestations. Ethiop J Health Sci [Internet]. 2016 [Citado 6 Sep 2020]; 26(2):187-92. Disponible en:

https://doi.org/10.4314/ejhs.v26i2.13

6. Shirley M. D., Tang H., Gallione C. J., Baugher J. D., Frelin L.P., et al. Sturge-Weber Syndrome and Port-Wine Stains Caused by Somatic Mutation in GNAQ. N Engl J Med [Internet]. 2013 [Citado 6 Sep 2020]; 368(21): 1971-79. Disponible en: https://doi.org/10.1056/NEJMoa1213507

7. Huang L., Couto J.A., Pinto A., Alexandrescu S., Madsen J. R., et al. Somatic GNAQ mutation is enriched in brain endothelial cells in Sturge-Weber Syndrome. Pediatr Neurol [Internet]. 2017 [Citado 6 Sep 2020]; 67: 59-63. Disponible en: https://doi. org/10.1016/j.pediatrneurol.2016.10.010

8. Aktories, K., Gierschik, P., Heringdorf D.M.z., Schmidt M., Schultz G., et al. cAMP guided his way: a life for $G$ protein-mediated signal transduction and molecular pharmacologytribute to Karl H. Jakobs. Naunyn-Schmiedeberg's
Arch Pharmacol. [Internet]. 2019 [Citado 6 Sep 2020]; 392: 887-911. Disponible en:

https://doi.org/10.1007/s00210-019-01650-1

9. He R., Liao S., Yao X., Huang R., Zeng J.,et al. Klippel-Trenaunay and Sturge-Weber Overlap Syndrome with KRAS and GNAQ mutations. Ann Clin Transl Neurol [Internet] 2020 [Citado 6 Sep 2020]; 7(7): 1258-64. Disponible en:

https://doi.org/10.1002/acn3.51106

10. Hildebrand M.S., Harvey A.S., Malone S., A. Damiano J.A., et al. Somatic GNAQ mutation in the forme fruste of Sturge-Weber syndrome. Neurol Genet [Internet]. 2018 [Citado 6 Sep 2020]; 4(3): e236. Disponible en:

https://doi.org/10.1212/NXG.0000000000000236 11. Nguyen V., Hochman M., Mihm M.C., Nelson J.S., Tan W. The Pathogenesis of Port Wine Stain and Sturge Weber Syndrome: Complex Interactions between Genetic Alterations and Aberrant MAPK and PI3K Activation. Int J Mol Sci [Internet]. 2019 [Citado 6 Sep 2020]; 20(9): 
2243. Disponible en: https://doi.org/10.3390/ ijms 20092243

12. Gao L., Yin R., Wang H., Guo, W., Song, W., et al. Ultrastructural Characterization of Hyperactive Endothelial Cells, Pericytes and Fibroblasts in Hypertrophic and Nodular Port Wine Stain Lesions. Br. J. Dermatol [Internet]. 2017 [Citado 7 Sep 2020]; 177(4): e105-e108. Disponible en: https://doi.org/10.1111/bjd.15373

13. Li W., Mukouyama Y.S. Tissue-specific venous expression of the $\mathrm{EPH}$ family receptor EphB1 in the skin vasculature. Dev Dyn [Internet]. 2013 [Citado 7 Sep 2020]; 242(8): 976-88. . Disponible en: https://doi.org/10.1002/dvdy.23985

14. Juhász C., Hu J., Xuan Y., Chugani H.T. Imaging increased glutamate in children with SturgeWeber syndrome: Association with epilepsy severity. Epilepsy Res [Internet]. 2016 [Citado 7 Sep 2020]; 122: 66-72. Disponible en: https://doi. org/10.1016/j.eplepsyres.2016.02.010

15. Stafstrom C.E., Staedtke V., Comi A.M.Epilepsy Mechanisms in Neurocutaneous Disorders: Tuberous Sclerosis Complex, Neurofibromatosis Type 1, and Sturge-Weber Syndrome. Front Neurol [Internet]. 2017 [Citado 7 Sep 2020]; 8: 87. Disponible en:

https://doi.org/10.3389/fneur.2017.00087

16. Formisano M., Abdolrahimzadeh B., Mollo R., Bruni P., Malagola R., et al. Billateral diffuse choroidal hemangioma in Sturge Weber syndrome: A case report highlighting the role of multimodal imaging and a brief review of the literature. J Curr Ophthalmol [Internet]. 2019 [Citado 7 Sep 2020]; 31(2): 242-49. Disponible en:

https://doi.org/10.1016/j.joco.2018.10.001

17. Gill N.C., Bhaskar N. Sturge - Weber syndrome: A case report. Contemp Clin Dent [Internet]. 2010 [Citado 7 Sep 2020]; 1(3): 183-85. Disponible en: https://doi.org/10.4103/0976-237X.72789

18. Waelchli R., Aylett S.E., Robinson K., Chong W.K., Martinez A.E., Kinsler V.A. New vascular classification of port-wine stains: improving prediction of Sturge-Weber risk. Br J Dermatol [Internet]. 2014 [Citado 7 Sep 2020]; 171(4):
861-67. Disponible en: https://doi.org/10.1111/ bjd. 13203

19. Lee J. W., Chung H. Y., Cerrati E.W., March O.T.M., Waner M. The Natural History of Soft Tissue Hypertrophy, Bony Hypertrophy, and Nodule Formation in Patients with Untreated Head and Neck Capillary Malformations. Dermatologic Surgery [Internet]. 2015 [Citado 9 Sep 2020]; 41(11), 1241-45. Disponible en:

https://doi.org/10.1097/DSS.0000000000000525

20. Harmon K.A., Day A.M., Hammill A.M., Pinto A.L., McCulloch C.E., et al. Quality of Life in Children With Sturge-Weber Syndrome. Pediatr Neurol [Internet]. 2019 [Citado 9 Sep 2020]; 101:26-32. Disponible en: https://doi. org/10.1016/j.pediatrneurol.2019.04.004

21. Junttila T.L., Alberto N., Winkels M., Greenwood M.D. Successful Reduction of Intraocular Pressure in a Patient with Glaucoma Secondary to Sturge-Weber Syndrome Using a Suprachoroidal Shunt. J Curr Glaucoma Pract [Internet]. 2020 [Citado 9 Sep 2020]; 14(1): 43-6. Disponible en:

https://doi.org/10.5005/jp-journals-10078-1266

22. Miller R.S., Ball K.L., Comi A.M., Germain-Lee E.L. Growth hormone deficiency in Sturge-Weber syndrome. Archives of Disease in Childhood [Internet]. 2006 [Citado 9 Sep 2020]; 91(4): 34041. Disponible en:

https://doi.org/10.1136/adc.2005.082578

23. Bachur C.D., Comi A.M., Germain-Lee E.L. Partial Hypopituitarism in Patients With Sturge-Weber Syndrome. Pediatric Neurology [Internet]. 2015 [Citado 9 Sep 2020]; 53(3), e5e6. Disponible en: https://doi.org/10.1016/j. pediatrneurol.2015.04.005

24. Pasquini L., Tortora D., Manunza F., Rossi Espagnet M.C., et al. Asymetric cavernous sinus enlargement: a novel finding in Sturge-Weber syndrome. Neuroradiol J [Internet]. 2019 [Citado 9 Sep 2020]; 61: 595-602. Disponible en: https:// doi.org/10.1007/s00234-019-02182-4

25. Da Silva T.J., Oliveira D.H., Moura I., Medeiros L., et al. Importance of GLUT1 in differential diagnosis of vascular anomalies. J Vasc Bras [Internet]. 2015 [Citado 9 Sep 2020]; 14(2): 16876. Disponible en:

https://doi.org/10.1590/1677-5449.0069

26. Comi A. Current Therapeutic Options in Sturge-Weber Syndrome. Semin Pediatr Neurol [Internet]. 2015 [Citado 10 Sep 2020]; 22(4): 295-301. Disponible en: https://doi.org/10.1016/j. spen.2015.10.005

27. Lipner S. Topical Adjuncts to Pulsed Dye Laser for Treatment of Port Wine Stains: Review of the Literature. Dermatol Surg [Internet]. 2018 [Citado 10 Sep 2020]; 44(6):796-802. Disponible en:

https://doi.org/10.1097/DSS.0000000000001507

28. Greveling K., Prens E.P., Van Doorn M.B. Treatment of port wine stains using Pulsing Dye Laser, Erbium YAG Laser, and topical rapamicin (sirolimus) - A randomized controlled trial. Laser Surg Med [Internet]. 2016 [Citado 10 Sep 2020]; 49(1): 104-9. Disponible en:

https://www.cochranelibrary.com/es/central/ doi/10.1002/central/CN-01341087/full

https://doi.org/10.1002/lsm.22548

29. Kaplan E.H., Kossoff E.H., Bachur C.D. Gholston M., Hahn J., et al. Anticonvulsant Efficacy in Sturge-Weber Syndrome. Pediatr Neurol [Internet]. 2016 [Citado 11 Sep 2020]; 58: 31-6. Disponible en: https://doi.org/10.1016/j. pediatrneurol.2015.10.015

30. Day A.M., Hammill A.M., Juhász C., Pinto A.L., Roach E.S., et al. Hypothesis: Presymptomatic treatment of Sturge-Weber Syndrome With Aspirin and Antiepileptic Drugs May Delay Seizure Onset. Pediatr Neurol [Internet]. 2019 [Citado 11 Sep 2020]; 90: 8-12. Disponible en: https://doi.org/10.1016/j. pediatrneurol.2018.04.009

31. Gómez-Cerdas M. Síndrome Neurocutáneo: Sturge Weber. Cron Cient [Internet]. 2019 [Citado 11 Sep 2020]; 12(12): 26-35. Disponible en: https://www.cronicascientificas.com/ index.php/ediciones/edicion-xii-mayo-agosto2019/26-ediciones/240-sindrome-neurocutaneosturge-weber 